\title{
Development of a standard of care for patients with valosin-containing protein associated multisystem proteinopathy
}

\author{
Manisha Korb ${ }^{1 *} \mathbb{D}$, Allison Peck ${ }^{4}$, Lindsay N. Alfano ${ }^{5}$, Kenneth I. Berger ${ }^{6}$, Meredith K. James ${ }^{7}$, Nupur Ghoshal ${ }^{8}$, \\ Elise Healzer ${ }^{9}$, Claire Henchcliffe', Shaida Khan ${ }^{10}$, Pradeep P. A. Mammen ${ }^{11}$, Sujata Patel ${ }^{12}$, Gerald Pfeffer ${ }^{13}$, \\ Stuart H. Ralston ${ }^{14}$, Bhaskar Roy ${ }^{15}$, William W. Seeley ${ }^{16}$, Andrea Swenson ${ }^{17}$, Tahseen Mozaffar ${ }^{1,3}$, \\ Conrad Weihl ${ }^{18,19}$ and Virginia Kimonis ${ }^{2,3}$ on behalf of the VCP Standards of Care Working Group
}

\begin{abstract}
Valosin-containing protein (VCP) associated multisystem proteinopathy (MSP) is a rare inherited disorder that may result in multisystem involvement of varying phenotypes including inclusion body myopathy, Paget's disease of bone (PDB), frontotemporal dementia (FTD), parkinsonism, and amyotrophic lateral sclerosis (ALS), among others. An international multidisciplinary consortium of 40+ experts in neuromuscular disease, dementia, movement disorders, psychology, cardiology, pulmonology, physical therapy, occupational therapy, speech and language pathology, nutrition, genetics, integrative medicine, and endocrinology were convened by the patient advocacy organization, Cure VCP Disease, in December 2020 to develop a standard of care for this heterogeneous and under-diagnosed disease. To achieve this goal, working groups collaborated to generate expert consensus recommendations in 10 key areas: genetic diagnosis, myopathy, FTD, PDB, ALS, Charcot Marie Tooth disease (CMT), parkinsonism, cardiomyopathy, pulmonology, supportive therapies, nutrition and supplements, and mental health. In April 2021, facilitated discussion of each working group's conclusions with consensus building techniques enabled final agreement on the proposed standard of care for VCP patients. Timely referral to a specialty neuromuscular center is recommended to aid in efficient diagnosis of VCP MSP via single-gene testing in the case of a known familial VCP variant, or multi-gene panel sequencing in undifferentiated cases. Additionally, regular and ongoing multidisciplinary team follow up is essential for proactive screening and management of secondary complications. The goal of our consortium is to raise awareness of VCP MSP, expedite the time to accurate diagnosis, define gaps and inequities in patient care, initiate appropriate pharmacotherapies and supportive therapies for optimal management, and elevate the recommended best practices guidelines for multidisciplinary care internationally.
\end{abstract}

\footnotetext{
*Correspondence: mkak@uci.edu

${ }^{1}$ Department of Neurology, University of California - Irvine School of Medicine, Orange, CA, USA

Full list of author information is available at the end of the article Collaborators: members of the VCP Standards of Care Working Group are listed in Additional file 1 (named authors) and Additional file 2 (coinvestigator and acknowledgements).
}

\begin{abstract}
Introduction
Valosin-containing protein (VCP) associated multisystem proteinopathy (MSP) is a rare and heterogeneous genetic disorder that can cause adult-onset inclusion body myopathy, Paget's disease of bone (PDB), and frontotemporal dementia (FTD), but other associated manifestations include parkinsonism, amyotrophic lateral sclerosis (ALS), neuropathy, spastic paraplegia, and cardiomyopathy, among others (Table 1 ). The previously
\end{abstract} original author(s) and the source, provide a link to the Creative Commons licence, and indicate if changes were made. The images or other third party material in this article are included in the article's Creative Commons licence, unless indicated otherwise in a credit line to the material. If material is not included in the article's Creative Commons licence and your intended use is not permitted by statutory regulation or exceeds the permitted use, you will need to obtain permission directly from the copyright holder. To view a copy of this licence, visit http://creativecommons.org/licenses/by/4.0/. The Creative Commons Public Domain Dedication waiver (http://creativeco mmons.org/publicdomain/zero/1.0/) applies to the data made available in this article, unless otherwise stated in a credit line to the data. 
Table 1 Clinical manifestations of VCP MSP

\begin{tabular}{|c|c|c|c|}
\hline Phenotype & System affected & Clinical features & Frequency in VCP patients \\
\hline Inclusion body myopathy & Muscle & $\begin{array}{l}\text { Axial and proximal weakness progressing } \\
\text { distally is most common, although presen- } \\
\text { tations resembling facioscapulohumeral } \\
\text { muscular dystrophy, oculopharyngeal } \\
\text { muscular dystrophy, and distal myopathy } \\
\text { have been described }\end{array}$ & $\sim 90 \%$ \\
\hline Paget disease of bone (PDB) & Skeletal & $\begin{array}{l}\text { Bone pain, bone deformities, pathological } \\
\text { fractures, hearing loss }\end{array}$ & $\sim 40 \%$ \\
\hline Frontotemporal dementia (FTD) & Cognitive & $\begin{array}{l}\text { Rapidly progressive behavioral impairment, } \\
\text { executive dysfunction, language impair- } \\
\text { ment. Often associated with Parkinsonian } \\
\text { features such as dystonia, tremor, gait } \\
\text { disturbance }\end{array}$ & $\sim 30 \%$ \\
\hline Respiratory dysfunction & Pulmonary & $\begin{array}{l}\text { Recurrent respiratory infections, weak } \\
\text { cough, aspiration, sleep disordered breath- } \\
\text { ing, respiratory failure }\end{array}$ & $40-50 \%$ \\
\hline Amyotrophic lateral sclerosis (ALS) & Upper and lower motor neurons & $\begin{array}{l}\text { Multifocal weakness, hyperreflexia and/ } \\
\text { or areflexia, atrophy, fasciculations, bulbar } \\
\text { weakness, respiratory muscle involvement, } \\
\text { weight loss }\end{array}$ & $\sim 10 \%$ \\
\hline Parkinson disease (PD) & Central nervous system & $\begin{array}{l}\text { Hypokinetic movement disorder, autonomic } \\
\text { dysfunction, various non-motor features }\end{array}$ & $4 \%$ \\
\hline Alzheimer disease (AD) & Cognitive & $\begin{array}{l}\text { Dementia with predominant amnestic and } \\
\text { higher order cognitive dysfunction }\end{array}$ & $2 \%[4]$ \\
\hline Spastic paraplegia & Upper motor neurons & $\begin{array}{l}\text { Length-dependent weakness, hyperreflexia, } \\
\text { spasticity, clonus }\end{array}$ & Isolated cases \\
\hline $\begin{array}{l}\text { Sensorimotor neuropathy (axonal } \\
\text { Charcot Marie Tooth disease } \\
(\text { CMT)) }\end{array}$ & Peripheral nerves & $\begin{array}{l}\text { Length-dependent weakness, muscle atro- } \\
\text { phy and sensory loss. Trophic foot changes } \\
\text { and distal areflexia }\end{array}$ & Isolated cases \\
\hline Cardiomyopathy & Cardiac & Exertional shortness of breath, heart failure & Uncertain. Reported in case series \\
\hline Dysphagia and dysarthria & Bulbar dysfunction & $\begin{array}{l}\text { Impaired swallowing function, reduced } \\
\text { speech volume and intelligibility }\end{array}$ & Uncertain \\
\hline Urinary and anal incontinence & Genitourinary, gastrointestinal & $\begin{array}{l}\text { Urinary incontinence, anal incontinence or } \\
\text { dysfunction }\end{array}$ & Uncertain \\
\hline
\end{tabular}

used term, inclusion body myopathy associated with Paget's disease of bone (and frontotemporal dementia (IBMPFD), is no longer favored given the other phenotypes that may occur. Other genes that are associated with a similarly presenting MSP syndrome include heterogeneous nuclear ribonucleoprotein $\mathrm{A} 2 \mathrm{~B} 1$ and $\mathrm{A} 1$ (hnRNPA2B1, hnRNPA1), sequestosome 1 (SQSTM1), matrin 3 (MATR3), T-cell restricted intracellular antigen 1 (TIA1), optineurin (OPTN), annexin 11 (ANXA11), and profilin 1 (PFN1I), many of which share common pathophysiology of disruption of RNA stress granule function or autophagic degradation, and patients presenting with these MSP syndromes may benefit from similar diagnostic and treatment strategies $[1,2]$.

On April 9, 2021, over 40 specialists attended a virtual international meeting to establish a consensus on standard of care recommendations for the diagnosis and treatment of VCP MSP. The meeting was organized by the patient advocacy organization, Cure VCP Disease, and multidisciplinary physicians and therapists presented on the clinical features, diagnosis, surveillance, and management of the different aspects of the disease. The topics discussed were genetic diagnosis, myopathy, FTD, PDB, ALS, Charcot Marie Tooth disease CMT, Parkinson's disease (PD)/parkinsonism, cardiomyopathy, respiratory dysfunction, supportive therapies [including physical and occupational therapy (PT/OT), speech-language pathology (SLP)], mental health, supplements, and nutrition. The goal was to develop a robust consensus-based set of recommendations from our experts based on systematic literature review and clinical experience; the recommendations for each sub-category of the disease are highlighted in Table 2. Due to a lack of evidence in VCP disease, we deferred to guidance and experience from diseases with phenotypic similarities to aspects of VCP, consistent with Level III evidence based on the Canadian Task Force on the Periodic Health Examination's Level of Evidence [3]. This report will summarize the recommendations from the meeting, with the aim of increasing awareness of VCP MSP, expediting time to accurate 


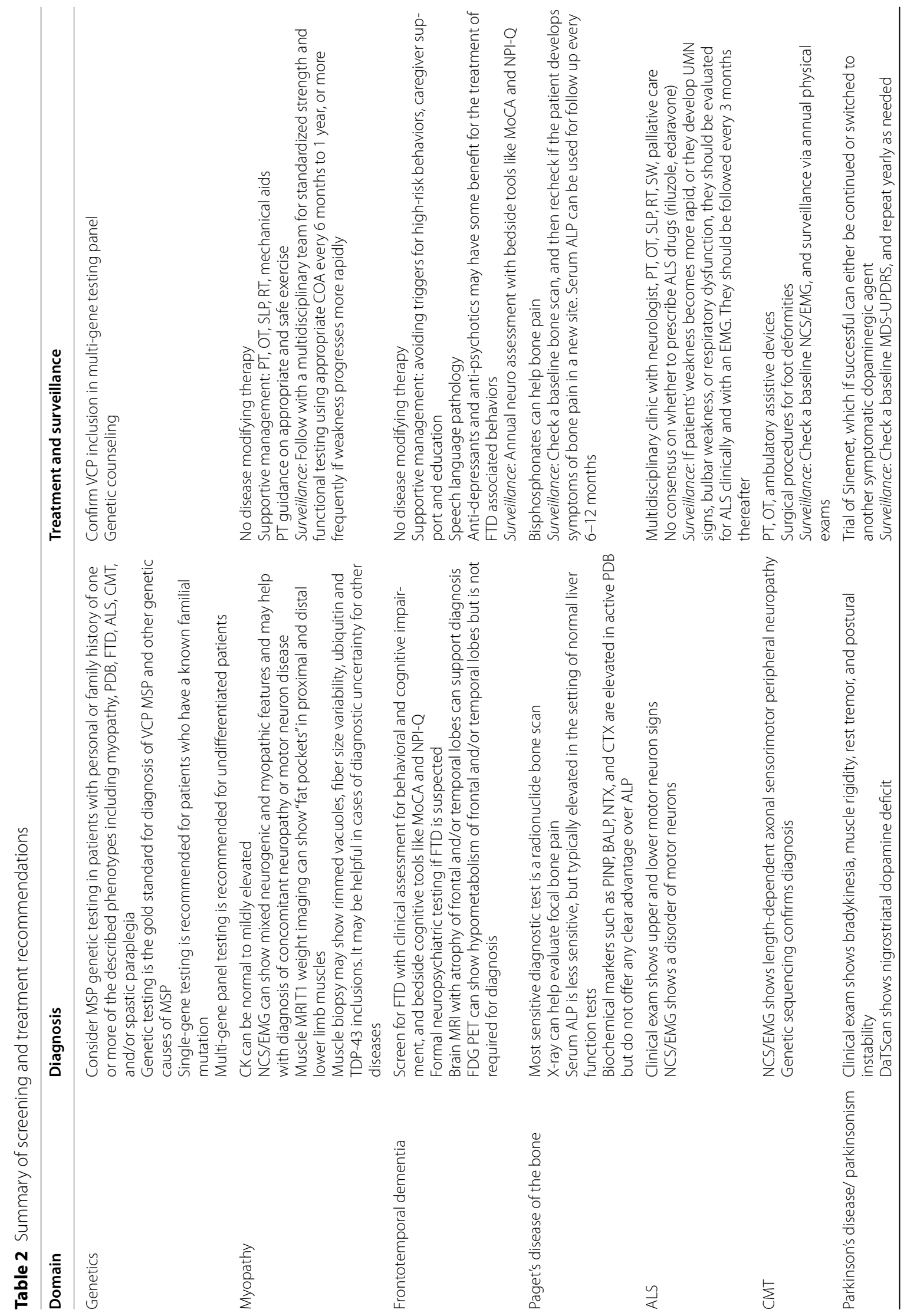




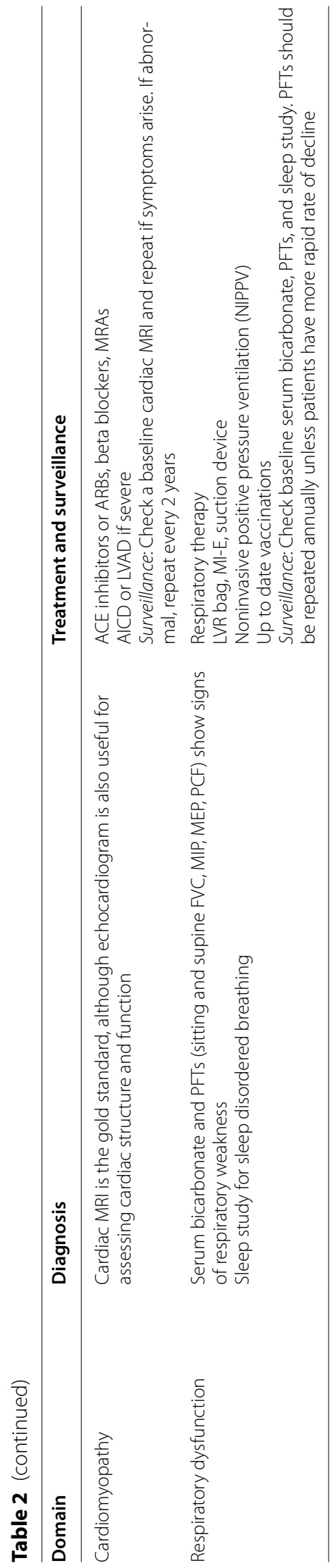


diagnosis, and establishing a multidisciplinary standard of care for appropriate pharmacotherapies and supportive therapies.

\section{Genetics}

VCP is a ubiquitously expressed and abundant ATPase involved with protein degradation and autophagy [5]. VCP mediates ubiquitin-dependent cellular processes through the ubiquitin-proteasome system (UPS), protein quality control, regulation of autophagy, transcription factor processing, membrane fusion, and cellular signaling [6]. Over 50 heterozygous missense mutations in $V C P$, mapped to chromosome 9p13.3-12, cause autosomal dominant VCP MSP $[4,7,8]$.

VCP mutations are the most common in genetically diagnosed families with MSP and are designated MSP1 [1]. Other genes associated with MSP may cause a clinically indistinguishable phenotype. MSP2 and MSP3 are caused by mutations in HNRNPA2B1 and HNRNPA1, respectively [9]. MSP4 is caused by mutations in SQSTM1 [10]. In addition, mutations in MATR3 are linked to familial myopathy with ALS and have rarely been associated with FTD $[11,12]$, and may be considered MSP5 [1]. TIA1 is associated with Welander distal myopathy, ALS, and FTD [13]. OPTN mutations are another cause of familial ALS and ALS-FTD [14], and genetic variation in OPTN is a risk factor for development of PDB $[14,15]$. Most recently, mutations in ANXA11 have been associated with IBM with ALS/FTD and have been proposed to be categorized as MSP6 [2]. Missense mutations in PFN1 are associated with familial ALS whereas PFN1 haploinsufficiency is associated with early onset and polyostotic PDB $[16,17]$. Disease caused by PFN1 mutations has recently been proposed as MSP7 [16, 18].

MSP should be considered in patients and families with one or more of the described phenotypes including myopathy, PDB, FTD, ALS, axonal CMT, and/or spastic paraplegia. Genetic testing is the primary and definitive means for diagnosis of VCP MSP and other genetic causes of MSP. Single-gene testing is recommended for patients who have a known familial mutation, while multi-gene panel testing is recommended for undifferentiated patients. Some multi-gene panels for myopathy, skeletal disorders, dementia, ALS, spastic paraplegia, neuropathy, and PD, include the VCP gene. However, older panels may not have included VCP as the phenotypic spectrum has expanded.

The identification of a pathogenic VCP variant confirms the diagnosis in a clinically affected individual or provides a pre-symptomatic diagnosis for an unaffected individual. The identification of a likely pathogenic variant may be considered diagnostic with the caveat that further evidence could result in later reclassification of the variant. If an unaffected individual has a positive result, there is a $90 \%$ chance that he or she will develop one or more of the features of VCP MSP by the age of 45 [19]. A variant of unknown significance (VUS) is a change in VCP that has not previously been associated with human disease; insufficient data exists to support whether the variant is benign or pathogenic, and therefore such a result needs further analysis as a possible new pathogenic variant before being considered as not clinically actionable. Detailed information regarding genetic variant interpretation and pathogenicity criteria may be found by referring to American College of Medical Genetics criteria [20].

The cardiac and respiratory complications of VCP MSP (see below) may benefit from early screening and management, and may be considered as part of the decision to pursue pre-symptomatic testing for VCP MSP. However presymptomatic testing may also be pursued to help make decisions regarding career, lifestyle, and reproductive planning [21]. Pre-symptomatic patients and patients with limited aspects of the MSP syndrome should be monitored by a healthcare professional on a regular basis to watch for development of clinical disease.

National guidelines vary, however genetic counseling should precede and follow pre-symptomatic genetic testing because of the significant implications including family planning, psychosocial counseling, and early identification and treatment of the varied aspects of this multifaceted disease. There can be psychological challenges encountered as a consequence of both positive and negative pre-symptomatic testing [22]. Genetic counselling follow-up should be offered for both mutation carriers and non-carriers, and clinicians should have awareness that additional mental health support may be of value in both situations [23]. Clinicians should be aware of challenges in genetic test result interpretation and genetic counseling, including the Genetic Non-Discrimination Act (GINA), and psychosocial considerations for both presymptomatic and symptomatic individuals.

\section{Myopathy \\ Clinical features}

Myopathy is the most common feature of VCP MSP affecting $90 \%$ of the individuals, with proximal predominant muscle weakness as well as frequent distal muscle involvement $[4,24,25]$. The mean age of onset is in the fifth decade $[4,24]$. Patients may develop foot drop, scapular winging, and scapuloperoneal weakness; facial weakness is less common [26]. Patients may become wheelchair-dependent, with loss of ambulation within $13 \pm 7$ years from symptom onset [26]. 


\section{Diagnosis}

Genetic testing remains the only definitive way to diagnose VCP myopathy. Other laboratory testing and imaging are generally nonspecific but can be supportive in the diagnosis of myopathy. Creatine kinase (CK) value can be normal or mildly elevated [24]. Nerve conduction studies (NCS) can be normal or in some cases show an axonal motor or sensorimotor neuropathy [27]. Electromyography (EMG) can show mixed neurogenic and myopathic features [27]. Electrodiagnostic studies may be helpful for ruling out other disease mimics and for identifying motor neuron disease. Muscle MRI T1 weight imaging has shown fatty infiltration, or "fat pockets," in both proximal and distal muscles of the lower limbs, with small areas of fat replacement surrounded by areas of normal muscle [28]. There is not enough data on Short TI inversion recovery (STIR) imaging, although in some isolated muscles it could show increased signal that may reflect inflammation [28].

Muscle biopsy can be done if there is diagnostic uncertainty and/or no genetic mutation is identified in genes associated with myopathies. It generally shows nonspecific changes, with fiber size variability, atrophic and hypertrophic fibers, and type 1 fiber predominance. While rimmed vacuoles are considered a hallmark of VCP myopathy, they are not always present and can be seen in other inherited muscle diseases [29, 30]. Congophilic inclusions, cytoplasmic ubiquitin, and TAR DNA-binding protein 43 (TDP-43) positive inclusions may be present [29].

\section{Treatment}

There is no approved disease modifying therapy for VCP myopathy, and the management is supportive. Several agents targeting the autophagy and VCP inhibition have shown some promise in preclinical studies [31-33]. Patients should be followed by a multidisciplinary clinic at least every 6 months; the components of this clinic should include a neurologist, PT, OT, SLP, respiratory therapy (RT), and social worker (SW). The team should manage the symptoms of limb and bulbar weakness, respiratory dysfunction, and muscle cramps. Other supportive measures, such as mechanical aids and weight control to avoid obesity can be beneficial, and these are described in more detail in the Supportive Therapies section of this article. For patients with imminent respiratory and cardiac failure, palliative care and end of life discussions should be initiated. Because VCP-specific data on treatment is lacking, we base this on the accepted approach to other vacuolar myopathies.

\section{Surveillance}

Patients with VCP mutations who do not currently have symptoms of myopathy, and family members who are at risk, should be screened for signs of muscle weakness via standardized strength and functional testing by a multidisciplinary team. If weakness exists, they should be followed clinically for signs of progression and possible intervention every 6 months to annually, or more frequently if progression is more rapid.

\section{Frontotemporal dementia \\ Clinical features}

FTD affects about $30 \%$ of patients with VCP mutations [4] and typically presents as the behavioral variant FTD (bvFTD) subtype [26], although other FTD syndromes such as forms of primary progressive aphasia (PPA) have been reported [34]. Manifestations depend on the clinical syndrome but may include social-emotional changes (apathy, disinhibition, loss of empathy, compulsive behaviors, and overeating) or language deficits (word-finding difficulties, loss of word comprehension, diminished fluency). The average age of onset of FTD in VCP patients is 55 years, which is earlier than in the general population [4].

\section{Diagnosis}

Diagnosis of FTD starts with a clinical history of typical behavioral impairment and/or language decline that results in a reduced level of functioning. In the presence of such a history, bedside tests like the Montreal Cognitive Assessment (MoCA) can be used to approximate the level of cognitive impairment [35], but such tests do not provide sensitive screening tests for FTD. The Neuropsychiatric Inventory Questionnaire (NPI-Q) is a structured, informant-based tool to ascertain some behavioral symptoms relevant to FTD. The MoCA and NPI-Q are preferred in general practice over more specialized, less accessible FTD screening tools, as the latter require training and experience; no screening tool has been validated for VCP-related FTD at this time. A concerning clinical history, with or without supportive evidence from brief cognitive/behavioral assessments, should prompt a brain MRI, screening dementia laboratory tests, and referral to a memory disorders clinic and/or neuropsychiatric testing, which can provide comprehensive assessment of behavioral and cognitive functioning. MRI is useful to rule out non-degenerative causes and may show frontal, insular, and/or anterior temporal lobe atrophy [36, 37].

Additional tests sometimes used in the assessment of patients with FTD include fluorodeoxyglucose (FDG)PET or amyloid-PET to rule out Alzheimer disease (AD), and lumbar puncture to rule out FTD mimics such as inflammatory or infectious diseases, or to rule out $\mathrm{AD}$ 
using CSF biomarkers for amyloid beta and phospho-tau [38].

\section{Treatment}

There is no disease modifying treatment for FTD, and we must draw on the standard of care for FTD in general as data on VCP-specific FTD treatment is lacking. Supportive measures such as caregiver education, re-direction techniques, removing triggers for highrisk behaviors, and establishing a daily routine can help improve a patient's quality of life. Caregiver support is essential, as caregivers are at high risk for burnout. Exercise and cognitive activity may have a protective effect, as an active lifestyle has been associated with slower functional decline in some forms of autosomal dominant FTD [39], although this has not been specifically tested in VCP FTD. Speech therapy may help improve communication skills via Augmentative and Alternative Communication (AAC), with a goal of delaying language decline [40].

Antidepressants such as selective serotonin reuptake inhibitors may help compulsive and overeating symptoms that can occur with FTD $[41,42]$. Antipsychotics, such as quetiapine or risperdone, or mood stabilizers such as valproate, may be indicated for disruptive aggressive or agitated behaviors, but these medications, which have serious side effects, should be prescribed under the direction of an expert familiar with pharmacological management of FTD. Drugs approved for the treatment of $\mathrm{AD}$, including rivastigmine, galantamine, donepezil, memantine, and the newly approved drug aducanumab, have either not been studied in FTD or have been proven ineffective [43, 44].

\section{Surveillance}

Symptomatic patients with $V C P$ mutations who lack FTD should undergo annual screening with a neurological assessment, informant interview, and bedside assessment tools like MoCA and NPI-Q. Among patients with a known mutation but no symptoms of any kind, surveillance may be calibrated to the patient's age and the estimated age-of-onset, based on affected members of the family.

\section{Paget's disease of the bone Clinical features}

PDB occurs in roughly $40 \%$ of patients with VCP MSP [4] and is characterized by focal abnormalities of bone remodeling due to an increase in osteoclastic bone resorption along with increased and disorganized bone formation. Affected bones can become enlarged and deformed, and this may result in bone pain, pathological fractures, secondary osteoarthritis, deafness, and nerve compression [45]. Some PDB patients may be asymptomatic, however, and disease may be found incidentally through lab or imaging studies [46].

\section{Diagnosis}

When a patient exhibits signs of PDB, the most sensitive diagnostic test is a radionuclide bone scan, which shows intense tracer uptake in affected bones. The bone scan should then be followed by X-ray of sites showing abnormal tracer uptake; X-ray can show cortical thickening, bone expansion, and bone deformity [47]. X-rays can help in evaluating the cause of focal bone pain and in this regard, physicians should remain cognizant of pain resulting from sources other than Paget's such as osteoarthritis.

In metabolically active PDB, serum alkaline phosphatase (ALP) is typically elevated in the setting of normal liver function tests. However, a normal ALP does not exclude a diagnosis of PDB, especially in early disease. Biochemical markers that reflect increased bone turnover such as procollagen type I intact $\mathrm{N}$-terminal propeptide (PINP), bone-specific alkaline phosphatase (BALP), crosslinked N-telopeptide of type I collagen (NTX), and collagen type 1 C-telopeptide (CTX) are elevated in active PDB but do not offer any clear advantage over ALP except in patients with chronic liver disease where liver enzymes may be elevated. Serum calcium, phosphate urea, and parathyroid hormone (PTH) are usually normal in PDB.

\section{Treatment}

Drawing on the standard of care for PDB in general rather than VCP-specific PDB given lack of available data, symptomatic PDB patients can be referred to a bone specialist-either an endocrinologist or rheumatologist. Bisphosphonates including zoledronic acid, pamidronate, risedronate and alendronic acid are first line treatments, and can help symptoms of bone pain [48]. Treatment courses can be repeated as needed if symptoms recur along with raised ALP or increased uptake on bone scan. Vitamin D deficiency should be corrected prior to administration of bisphosphonates, to mitigate against hypocalcemia [49]. If bisphosphonates are contraindicated, calcitonin may be considered for bone pain, but long-term use has been associated with an increased risk of certain cancers. It is unclear whether early intervention with bisphosphonates in VCP MSP who do not have PDB symptoms would be beneficial. The issue of whether prophylactic therapy is beneficial in early PDB is being investigated in the ZIPP study [ISRCTN11616770] where SQSTM1 mutation carriers (a gene implicated in MSP4) have been treated with zoledronic acid or placebo. This study is expected to report during 2022 [50]. 
Patients who have concomitant arthritis or nerve compression may require analgesic, neuropathic pain medication, or NSAID therapies. Orthopedic surgery can be required in up to $10 \%$ of $\mathrm{PDB}$ patients, most commonly for fracture repair, hip or knee arthroplasty, or spinal surgery for spinal stenosis [51].

\section{Surveillance}

For newly diagnosed VCP patients, physicians should check a baseline bone scan to assess disease extent. If the patient develops symptoms of bone pain at a site which was initially unaffected, the scan may be repeated. Serum ALP can be repeated every 6-12 months to assess metabolic activity. If ALP values are raised and accompanied by pain at an affected site this would be an indication for further testing and treatment.

\section{Amyotrophic lateral sclerosis Clinical features}

ALS is reported to occur in $10 \%$ of VCP patients [4], and is clinically indistinguishable from ALS resulting from other familial or sporadic causes [1]. It is a progressive neurodegenerative disorder that affects both upper and lower motor neurons (UMN, LMN), resulting in muscle atrophy, weakness, fasciculations, and brisk reflexes. Bulbar involvement is characterized by dysphagia and dysarthria. Death typically occurs as a result of respiratory failure.

\section{Diagnosis}

The diagnosis of ALS is based on diagnostic criteria, which has evolved over time [52-54]. Patients present with progressive weakness in bulbar, cervical, thoracic, or lumbar regions, and clinical findings of upper and lower motor neuron involvement [52]. EMG can be a supportive tool for confirming lower motor neuron dysfunction and assessing extent and severity of the disease. It is also helpful in diagnosis of cases of VCP myopathy that appear to be evolving into ALS, and when additional pathology like a compressive neuropathy needs to be evaluated. Serum CK may be normal or mildly increased, but typically does not exceed $1000 \mathrm{U} / \mathrm{L}$. VCP gene testing should be included in familial ALS gene panels any time genetic testing is being performed in ALS, since VCP mutations can be found in sporadic ALS patients.

\section{Treatment}

The mainstay of management of VCP patients with an ALS phenotype is regular follow up in a specialized multidisciplinary clinic on average every 3 months, as is done for ALS patients without VCP mutations. The components of this clinic include a neurologist, PT, OT, SLP, $\mathrm{RT}$, and SW. The team manages the symptoms of limb and bulbar weakness, sialorrhea, respiratory dysfunction, muscle cramps, spasticity, pseudobulbar affect, depression, and anxiety. Interventions include percutaneous endoscopic gastrostomy (PEG) placement, non-invasive positive-pressure ventilation (NIPPV), and rarely, tracheostomy with invasive mechanical ventilation. End-of-life decisions, hospice, and palliative care referrals are discussed with the multidisciplinary care team [55].

Although there is no cure for ALS, two medications, riluzole and edaravone, are FDA-approved for slowing disease progression. The decision to offer these drugs is based on provider and patient preference, as there is no evidence to support benefits or risks in VCP-specific ALS.

\section{Surveillance}

VCP patients should be assessed by a neurologist every 6 months for signs of progression of weakness. If rate of decline becomes more rapid and/or patients develop bulbar or respiratory dysfunction or UMN signs, ALS should be considered. An EMG can support a diagnosis of ALS. At this point, patients should be followed every 3 months in the multidisciplinary clinic.

\section{Charcot Marie Tooth disease \\ Clinical features}

CMT, also called hereditary motor and sensory neuropathy (HMSN), refers to familial neuropathies with extensive clinical and genetic heterogeneity. VCP MSP has been associated with autosomal dominant CMT (referred to as CMT2), with the designation CMT2Y (OMIM entry $616687)$, although reports of these cases remain rare [56, 57]. Outside of cases of CMT2Y, it is unknown if peripheral neuropathy is a coexisting feature of VCP MSP; it may, at times, be secondary to a separate condition such as diabetes.

CMT patients present with progressive length-dependent axonal loss in sensory and motor nerves, with predominant motor features. Weakness and atrophy occur in intrinsic foot muscles causing hammer toes and pes cavus, and the calves and intrinsic hand muscles can be involved as the disease progresses.

\section{Diagnosis}

CMT2Y is not clinically distinguishable from other forms of CMT2, so diagnosis must be achieved through genetic sequencing. An NCS/EMG shows axonal length-dependent sensorimotor neuropathy.

\section{Treatment}

VCP patients with a CMT2Y phenotype should receive supportive care with PT, OT, ambulatory assistive devices such as walking aids, ankle-foot orthotics (AFO) 
and hand splinting if necessary. Surgical procedures for foot deformities may be considered in some cases as well.

\section{Surveillance}

VCP patients should undergo baseline NCS/EMG at the time of diagnosis in the presence of suspicious clinical features to determine whether an axonal neuropathy is present, but surveillance testing is unlikely to be useful unless patients develop new symptoms of polyneuropathy or compressive neuropathy.

\section{Parkinson's disease/parkinsonism Clinical features}

Four percent of VCP patients have parkinsonism [4], and some patients are diagnosed with PD. Intrafamilial variability in phenotype has been well described for VCP patients and in some includes PD/parkinsonism [58]. Additionally, a link is supported by the finding of a greater prevalence of $\mathrm{PD} /$ parkinsonism in those with VCP mutations [4].

Clinical manifestations of PD and parkinsonism include bradykinesia, muscle rigidity, rest tremor, and postural instability [59]. Other motor features may include hypophonia, hypomimia, slowed and uncoordinated movement, stiffness and cramps, dystonia, imbalance, and dysphagia. Non-motor features may include hyposmia, mood disorders and anxiety, rapid eye movement (REM) sleep behavior disorders, autonomic dysfunction, hallucinations, mild cognitive impairment, and dementia.

\section{Diagnosis}

PD and parkinsonism are diagnosed based upon neurological history and exam. Neuroimaging biomarkers reflecting nigrostriatal dopamine deficit include 123I-ioflupane SPECT (DaTscan) or other dopamine transporter radioligands detected by SPECT or PET, and FDOPAPET. Genetic testing should be considered in PD if young onset, atypical features, or family history, and the presence of other manifestations of VCP MCP such as Paget's disease, ALS, or other should prompt VCP testing.

\section{Treatment}

Given the lack of data on VCP-specific PD treatment, we recommend standard of care PD treatment for these patients. Treatment response to carbidopa-levodopa for parkinsonism remains the gold standard, although multiple other medications may be advised based upon specific symptoms. If this medication improves symptoms, it can either be continued or switched to alternative symptomatic agents. There is no known case of DBS or other surgical treatment for such patients with VCP mutations to our knowledge in the literature.

\section{Surveillance}

In newly diagnosed VCP patients without significant signs of parkinsonism, it is reasonable to perform regular screening using clinical batteries in a multidisciplinary clinic, possibly yearly. The most important clinical battery tool is the Movement Disorder Society-Unified Parkinson's Disease Rating Scale (MDS-UPDRS), but other non-motor symptoms and signs are also informative. A remaining major challenge is how to discern whether such rating scales are affected by other manifestations of VCP MSP, such as myopathy.

\section{Cardiomyopathy Clinical features}

The prevalence of cardiomyopathy among patients with VCP MSP is unknown but appears to be rare $[60,61]$. VCP patients without extensive cardiac involvement may be asymptomatic, but as cardiomyopathy progresses to systolic dysfunction with increasing left ventricular dilation and pressure overload, they are more likely to become symptomatic. Patients with left ventricular failure may develop dyspnea with exercise intolerance. Physical exam findings may reveal signs of a volume overloaded state including elevated jugular venous pressure, hepatic congestion, pleural effusion with crackles, and lower extremity edema. Respiratory and cardiac failure can lead to death between the ages of $40 \mathrm{~s}$ to $60 \mathrm{~s}$ [62]. A recent study showed a higher prevalence of diastolic dysfunction in $\mathrm{VCP}$ patients indicating it may be an early sign of cardiac involvement [63]. It is important to exclude other etiologies of cardiomyopathy or congestive heart failure such as ischemic heart disease.

\section{Diagnosis}

Echocardiogram is useful to assess cardiac function and structure when diagnosing cardiomyopathy. However, cardiac MRI is now the gold standard for obtaining accurate cardiac dimensions and measuring function in most neuromuscular disorders because it is not hindered by anatomic abnormalities such as kyphosis [61].

\section{Treatment}

In patients with suspected or documented heart failure, a referral to a specialist is appropriate. Heart failure medications improve cardiac parameters in neuromuscular patients with non-ischemic cardiomyopathy. The three most useful classes of drugs for reversing cardiac remodeling related to cardiomyopathy are angiotensin-converting enzyme (ACE) inhibitors or angiotensin-receptor blockers (ARBs), beta-adrenergic receptor blockers, and mineralocorticoid receptor antagonists (MRAs). Other guideline directed medical therapies for heart failure (i.e. 
digoxin, diuretics, and others) have also been shown to reduce morbidity and mortality in patients with nonischemic cardiomyopathies with a reduced ejection fraction [61].

If cardiomyopathy progresses despite aggressive pharmacotherapies, other treatment considerations include implantation of an automated implantable cardioverterdefibrillator (AICD) with or without biventricular pacemaker, and implantable left ventricular assist devices (LVADs).

\section{Surveillance}

Given the possibility that VCP patients with cardiomyopathy may be asymptomatic, it is reasonable to perform a cardiac MRI at baseline to determine present cardiac function. VCP patients should be monitored for symptoms of cardiomyopathy annually, and a repeat cardiac MRI can be performed if abnormal symptoms or clinical signs develop. For patients with detectable cardiac involvement on cardiac MRI such as delayed gadolinium enhancement in the ventricular wall or reduced ejection fraction, cardiac MRI should be repeated every 2 years to evaluate for cardiac remodeling following pharmacologic interventions.

\section{Respiratory dysfunction Clinical features}

Respiratory muscle weakness occurs in at least $40-50 \%$ of patients with VCP MSP [64, 65]. Early manifestations of respiratory involvement may include recurrent tracheobronchitis and pneumonia due to weakness of the expiratory muscles with consequent impairment of cough strength and efficacy [66]. Progressive weakening of respiratory muscles leads to development of sleep disordered breathing characterized by nocturnal hypoventilation and oxygen desaturation [67]. In addition, patients with bulbar symptoms are at increased risk for aspiration. Ultimately, if not properly managed, these changes can result in respiratory failure and daytime alveolar hypoventilation (i.e. hypercapnia). Recognition and treatment of respiratory failure is critical since it is a frequent cause of death in VCP patients $[24,68]$.

\section{Diagnosis}

Respiratory muscle function can be assessed using pulmonary function tests [PFTs, particularly forced vital capacity (FVC): sitting and supine], maximum inspiratory and expiratory muscle pressures (MIP and MEP), and peak cough flow (PCF). Sleep disordered breathing and nocturnal hypoventilation can be identified by either a full in-lab nocturnal polysomnography or limited at-home overnight monitoring. The presence of respiratory failure and hypercapnia is often assessed using arterial blood gas analysis, but this test may not be readily available in some outpatient settings. Alternatively, measurement of the serum bicarbonate in venous blood provides an easily obtainable and reliable marker of renal compensation for chronic carbon dioxide retention.

Bulbar and cognitive dysfunction increase risk for pulmonary complications, and limit ability to monitor patients with PFTs and sleep studies.

\section{Treatment}

Extrapolating from management of respiratory dysfunction in other neuromuscular disorders, the treatment team should include a physical therapist, respiratory therapist, and a pulmonary specialist with expertise in management of patients with neuromuscular disease. The patient and their caregivers should maintain up to date vaccinations. Lung volume recruitment (LVR) and cough augmentation is important for the clearance of secretions and can be facilitated with LVR bag, mechanical insufflation/exsufflation (MI-E), and suction devices as needed. Respiratory infections should be treated early and aggressively. Non-invasive positive pressure ventilation (NIPPV), such as bilevel positive airway pressure (BiPAP) or volume assured pressure support (VAPS), should be initiated at the onset of nocturnal hypoventilation or with deterioration of respiratory function (e.g. arterial $\mathrm{PCO}_{2}$ $>45 \mathrm{mmHg}$, FVC $<50 \%$ of predicted, $\mathrm{MIP}<-60 \mathrm{~cm}$ $\mathrm{H}_{2} \mathrm{O}$ ). Therapy with continuous positive airway pressure (CPAP) should be reserved for individuals that demonstrate obstructive sleep apnea in the absence of nocturnal hypoventilation. The role of respiratory muscle training to improve clinical outcomes is unclear and is currently being investigated in VCP MSP (curevcp.org).

Supplemental oxygen and/or therapy with respiratory depressants (e.g. sleep aids, narcotics, anxiolytics and antidepressants) should be withheld if possible as they lower central respiratory drive and can induce or worsen hypercapnia. Diuretic therapy may be used to reverse peripheral edema in patients with cor pulmonale. However, primary metabolic alkalosis with impaired central respiratory drive may ensue because of the associated chloride and potassium deficiency.

\section{Surveillance}

Serum bicarbonate and PFTs can be checked annually in myopathy phenotype if stable, or every 3-6 months if deteriorating and/or ALS phenotype. A sleep study should be performed at baseline in patients with documented or symptomatic weakness. 


\section{Supportive therapies (physical and occupational therapy, speech-language pathology, and respiratory therapy)}

VCP MSP patients should have access to an individualized, comprehensive care plan with an interdisciplinary team including physical therapy (PT), occupational therapy (OT), speech language pathology (SLP), and respiratory therapy (RT). The impacts of VCP MSP across body structures and function should be routinely monitored and managed to optimize a patient's independence with activities of daily living (ADLs) and quality of life (QOL). Clinical outcome assessments (COA) performed by trained practitioners are useful for assessing function and to track the level of independence and/or assistance required to complete ADLs. Use of standardized COAs also facilitate proactive planning for procurement of any needed assistive devices, home and work modifications, or other supports. Including a patient-reported outcome (PRO) measure with strong psychometric properties is also beneficial for assessing health indices and QOL and is recommended to be completed at least annually. Pain and fatigue PROs should also be monitored at least annually with quantitative severity scales as these may impact both physical and cognitive function. Standardized tools are preferred over generalized scales.

\section{Physical and occupational therapy}

Physical and occupational therapy focus on helping patients maintain or adapt to changes in their function and ADLs to promote independence and QOL. While surveillance by PT and OT is recommended during multidisciplinary clinic visits, if there is difficulty with performing ADLs, mobility, or transfers, patients should be referred to PT and OT for ongoing treatment including appropriate exercises, home adaptations, and/or equipment. Regular and monitored aerobic exercise programs of low to moderate intensity are recommended to preserve function, strength, range of movement, endurance, balance, independence with ADLs, and participation [69, 70]. Inactivity can lead to further weakness and functional decline [71], so maintenance of current strength versus targeted muscle strengthening with proper monitoring may be indicated in some patients. While exercise is recommended there remains a lack of evidence for the most effective prescription in VCP MSP. Relevant assistive devices include ankle foot orthotics, canes, walkers, wheelchairs, chair seat risers, mobile arm supports, and hoists/ lift systems.

\section{Speech-language pathology}

The assessment of bulbar function is crucial and closely tied to respiratory function and nutritional status. SLP measures include swallowing function, speaking rate, overall speech intelligibility. A swallow study (e.g. videofluoroscopy or flexible endoscopy) can identify difficulties with swallowing permitting targeted treatment. An augmentative and alternative communication (AAC) examination can be conducted to ensure optimal communication, as well as provide education and training for using various external communication tools, like an alphabet board, tablet, or speech-generating device.

\section{Respiratory therapy}

RT is critical for monitoring and optimally managing respiratory function. PFTs should be performed at least annually, including measurements of FVC in sitting and supine positions. Chest physiotherapy, non-invasive ventilation, and insufflation-exsufflation devices should be initiated in a timely fashion.

\section{Mental health}

VCP MSP patients are at risk for higher levels of stress, depression, and anxiety due to the inherent rare nature of the disease, which predisposes them to misdiagnosis, differing clinical opinions, lack of resources and information, economic burden, and isolation [72]. Compounding all of these issues is the absence of true disease modifying therapies for the major components of the disease, aside from bisphosphonates for PDB. However, appropriate therapeutic support can help patients manage and process their diagnosis, work towards short- and long-term goals to improve their quality of life, engage in peer or professional support groups, facilitate challenging conversations with family members, reduce the risk of selfharm, and validate feelings of fear, grief, and anger.

Evidence-based practice includes screening all patients for behavioral health signs and symptoms and referring to behavioral providers upon learning of their diagnosis [73]. Collaboration between the multidisciplinary team and community mental health providers ensures a holistic approach to supporting individuals living with VCP, both at the time of diagnosis and throughout their experience with the disease.

\section{Urinary and anal incontinence}

VCP patients may experience urinary or anal incontinence/dysfunction as reported in isolated cases [26, 68], however is underrecognized. In other neuromuscular disorders, urinary and anal dysfunction are present and can significantly lower the quality of life for patients [74-76]. Screening for these symptoms during annual examinations through patient survey or interview may be appropriate and help to discern the etiology of the incontinence. Functional incontinence may require addressing issues of mobility, bathroom accessibility or scheduled 
voiding whereas other forms of incontinence may require referral to a specialist. Further studies are needed to determine the true prevalence of urinary and anal dysfunction in VCP patients.

\section{Supplements and nutrition}

Specific diets and supplements have not been formally studied in VCP patients. A lipid enriched diet was shown to improve survival, motor activity, muscle pathology and the autophagy cascade in $\mathrm{VCP} \mathrm{P}^{\mathrm{R} 155 \mathrm{H} / \mathrm{R} 155 \mathrm{H}}$ homozygous mice [77], however this requires further study in humans. Given the oxidative stress that occurs in VCP MSP pathophysiology, it is reasonable to recommend an anti-inflammatory diet that is rich in fruits and vegetables, whole grains, lean proteins, and fatty fish, and avoid highly processed foods and products that contain preservatives, pesticides, and artificial ingredients. Supplements that may play a role in reducing oxidative stress include $\mathrm{N}$-acetyl cysteine, alpha lipoic acid, acetyl-L-carnitine, vitamin B12, and coenzyme Q10, among others, although there remains a lack of evidence that these supplements alter symptoms or the course of the disease. In addition, there is evidence that a Mediterranean diet may offer protection in dementia and cardiovascular disease [78], which could be extrapolated with regards to VCP pathology, but further studies of this theory are needed. It is important to identify barriers to nutritional literacy and access to resources that may limit patients and caregivers understanding of dietary recommendations. A referral to a nutritionist or registered dietician may be appropriate.

\section{Conclusion}

VCP MSP is a rare disease with diverse multi-organ phenotypes that warrants better awareness and management in the physician community. Our international multidisciplinary meeting in April 2021 established the standard of care recommendations for the various aspects of the disease from all relevant specialties.

Development of an optimal clinical outcome toolbox for assessment of patients with VCP MSP is necessary to better monitor and manage patients. It may be challenging to recruit a large sample size at any particular institution, but multisite clinical trials using a spoke and hub model with centralized administration and scoring of COAs could improve results. Similarly, utilizing COAs that can be validly administered and scored within a patient home environment can help reduce the burden of travel to distant sites for trial visits. Future largescale studies are needed to better inform clinical practice guidelines on recommended treatments, therapies, and exercise programs, and to identify characteristics associated with responders to proposed interventions.

\section{Supplementary Information}

The online version contains supplementary material available at https://doi. org/10.1186/s13023-022-02172-5.

Additional file 1. Named authors.

Additional file 2. Co-investigators and acknowledgements.

\section{Acknowledgements}

VCP SOC Working Group Roberto Fanganiello PhD, Grace Lee MSc, Ryan Patrick Mahoney BSc, Jordi Diaz-Manera MD PhD, Teresinha Evangelista MD, Miriam Freimer MD, Thomas E Lloyd MD PhD, Benison Keung MD, Hani Kushlaf MD, Margherita Milone MD PhD, Merrilee Needham PhD MBBS FRACP, Johanna Palmio MD PhD, Tanya Stojkovic MD, Rocío-Nur Villar-Quiles MD PhD, Leo H. Wang MD PhD, Matthew P. Wicklund MD, Frederick R. Singer MD, Mallory Jones, Bruce L. Miller MD, S. Ahmad Sajjadi MD, Andre Obenaus PhD, Michael D Geschwind MD PhD, Ammar Al-Chalabi PhD MB ChB, James Wymer MD PhD, Nita Chen MD, Katie Kompoliti MD, Stephani C. Wang MD, Catherine A. Boissoneault PT (NO), Betsaida Cruz-Coble PT DPT NCS, Kendrea L. (Focht) Garand PhD CSCD CCC-SLP BCS-S CBIS CCRE, Anna J. Rinholen OTR/L, Lauren Tabor-Gray PhD CCC-SLP.

Additional Acknowledgements Jeffrey Rosenfeld PhD MD FAAN, Ming Guo MD $\mathrm{PhD}$, Nathan Peck MBA.

Please see Additional file 2 for details on the roles of each individual.

\section{Authors' contributions}

MK, VK participated in study concept and design; acquisition, analysis, and interpretation of data; drafting/revising the manuscript; critical revision of the manuscript for important intellectual content; study supervision. AP participated in study concept and design; acquisition, analysis, and interpretation of data; drafting/revising the manuscript. LA, KB, MJ, NG, EH, CH, SK, PM, SP, GP, SR, BR, WS, AS participated in acquisition, analysis, and interpretation of data; drafting/revising the manuscript; critical revision of the manuscript for important intellectual content. TM participated in study concept and design and study supervision. CW participated in study concept and design; acquisition, analysis, and interpretation of data; critical revision of the manuscript for important intellectual content. Please see Additional file 1 for additional information on each author. All authors read and approved the final manuscript.

\section{Funding}

This work was supported by the National Institute of Health (NIH

R01AR074457 awarded to TM) and the NIH funded UT Southwestern Senator Paul D. Wellstone Muscular Dystrophy Specialized Research Center Grant (NIH P50HD087351 awarded to PPAM).

\section{Availability of data and materials}

Data sharing not applicable to this article as no datasets were generated or analyzed during the current study.

\section{Declarations}

Ethics approval and consent to participate

This manuscript does not report on or involve the use of any animal or human data or tissue.

\section{Consent for publication}

This manuscript does not contain any individual persons' data.

\section{Competing interests}

MK has served on advisory boards for Biogen, Argenx, and CSL Behring, and on the speaker's bureau for Biogen. AP does not have any competing interests to disclose. LA reports grant support from Cure VCP Disease; provides consultancy services for training and quality control through ATOM International for the following companies: Amicus Therapeutics Pty Ltd, Catabasis, Genethon, Italfarmaco, NS Pharma, Pfizer, PTC Therapeutics; reports royalties and other support through Nationwide Children's Hospital from Sarepta Therapeutics; royalties for licensed technologies; other support through Nationwide Children's Hospital from Novartis Gene Therapies; advisory board for Biogen. KB 
has consulted for and/or served as a member of advisory boards for SanofiGenzyme, Inventiva Pharma, Amicus Therapeutics, Valerion Therapeutics, and Spark Therapeutics. MJ provides consultancy services for the following companies: ATOM International (covers consultancy services provided to Amicus Therapeutics Pty Ltd, Ascendis Pharma, Biomarin, Catabasis, Faraday, FibroGen, Genethon, Italfarmaco, NS Pharma, Pfizer, PTC Therapeutics, QED Therapeutics Ltd, Reveragen, Sarepta Therapeutics, outside the submitted work. MKJ has received payment for participation on Advisory Boards for F. Hoffman La Roche AG, PTC Therapeutics and fee support for PhD studies from the Jain Foundation, outside the submitted work. NG has participated or is currently participating in clinical trials of anti-dementia drugs sponsored by the following companies: Bristol Myers Squibb, Eli Lilly/Avid Radiopharmaceuticals, Janssen Immunotherapy, Novartis, Pfizer, Wyeth, SNIFF (The Study of Nasal Insulin to Fight Forgetfulness) study, and A4 (The Anti-Amyloid Treatment in Asymptomatic Alzheimer's Disease) trial. She receives research support from $\mathrm{NIH}$, Tau Consortium, and the Association for Frontotemporal Degeneration. EH does not have any competing interests to disclose. $\mathrm{CH}$ has served on advisory boards for Axent Biosciences Inc, Acorda Therapeutics, Amneal Pharmaceuticals, Mitsubishi Tanabe Pharma, and US WorldMeds, and as a consultant for Zywie Bio. SK has served on advisory boards for Alexion Pharmaceuticals, Inc, UCB Pharmaceuticals, Inc, Immunovant, Inc, and Argenx. PM declares the following competing interests: American Heart Association (member of the AHA Career Development Research Grant Committee), California Institute of Regenerative Medicine (member of the Grants Working Group), CareDx Inc. (Site PI for the SHORE Registry), Catabasis Inc. (research grant), Dyne Therapeutics (consultant and member of the DMD Scientific Advisory Board), National Institute of Health (research grants and ad hoc grant reviewer for the NIH SMEP and MOSS Study Sections), Novartis Gene Therapies (member of the Data Monitoring and Safety Committee), and PhaseBio Inc. (research grant and member of the Scientific Advisory Board). SP owns a wellness company for supplements called Wellness with Sujata. GP does not have any competing interests to disclose. SR does not have any competing interests to disclose. BR has served as a consultant for Alexion Pharmaceuticals. WS does not have any competing interests to disclose. AS receives research support from Amylyx Pharmaceuticals, Alexion Pharmaceuticals, ALS Association, Mass General Hospital, NIH/NINDS, and Cytokinetics Inc, and serves on an IDMC for Alexion. TM has served in an advisory capacity for Abbvie, Alexion, Amicus, Argenx, Audentes, Maze Therapeutics, Modis, Momenta, Ra Pharmaceuticals, Sanofi-Genzyme, Sarepta, Spark Therapeutics, UCB, and Ultragenyx. He serves on the speaker's bureau for Sanofi-Genzyme. Dr. Mozaffar serves on the medical advisory board for the Myositis Association, Neuromuscular Disease Foundation, Myasthenia Gravis Foundation of California and Myasthenia Gravis Foundation of America. Dr. Mozaffar receives research funding from the Myositis Association, the Muscular Dystrophy Association, the National Institutes for Health and from the following sponsors: Alexion, Amicus, Argenx, Audentes, Bristol-Myers-Squib, Cartesian Therapeutics, Grifols, Momenta, Ra Pharmaceuticals, Sanofi-Genzyme, Spark Therapeutics, UCB, and Valerion. He serves on the data safety monitoring board for Acceleron, Avexis, Sarepta, and the NIH. CW has served on advisory boards for Acceleron, Sarepta, Abata, and Orphazyme, and as a consultant for Sarepta. VK does not have any competing interests to disclose.

\section{Author details}

'Department of Neurology, University of California - Irvine School of Medicine, Orange, CA, USA. ${ }^{2}$ Department of Pediatrics, University of California - Irvine School of Medicine, Orange, CA, USA. ${ }^{3}$ Department of Pathology \& Laboratory Medicine, University of California - Irvine School of Medicine, Orange, CA, USA. ${ }^{4}$ Cure VCP Disease, Americus, GA, USA. ${ }^{5}$ The Abigail Wexner Research Institute at Nationwide Children's Hospital, Columbus, OH, USA. ${ }^{6}$ Department of Medicine (Pulmonary), NYU Grossman School of Medicine, New York, NY, USA. ${ }^{7}$ The John Walton Muscular Dystrophy Research Centre, Newcastle University and Newcastle Hospitals NHS Foundation Trust, Newcastle Upon Tyne, UK. ${ }^{8}$ Department of Neurology and Psychiatry, Washington University in St. Louis, St. Louis, MO, USA. ${ }^{9}$ Thriving Hope Consulting, Vinton, IA, USA. ${ }^{10}$ Department of Neurology \& Neurotherapeutics, University of Texas Southwestern Medical Center, Dallas, TX, USA. ${ }^{11}$ Department of Medicine (Cardiology), University of Texas Southwestern Medical Center, Dallas, TX, USA. ${ }^{12}$ Wellness with Sujata, Wadsworth, OH, USA. ${ }^{13}$ Hotchkiss Brain Institute, University of Calgary Cumming School of Medicine, Calgary, AB, Canada. ${ }^{14}$ Institute of Genetics and Cancer at the University of Edinburgh, Edinburgh, SCT, UK. ${ }^{15}$ Department of Neurology, Yale School of Medicine, New Haven, CT, USA. ${ }^{16}$ Weill Institute for Neurosciences, University of California San Francisco, San Francisco, CA, USA. ${ }^{17}$ Department of Neurology, University of lowa Hospitals and Clinics, lowa City, IA, USA. ${ }^{18}$ Department of Neurology, Washington University in St. Louis, St. Louis, MO, USA. ${ }^{19}$ The Hope Center, Washington University in St. Louis, St. Louis, MO, USA.

Received: 8 October 2021 Accepted: 16 January 2022

Published online: 29 January 2022

\section{References}

1. Korb MK, Kimonis VE, Mozaffar T. Multisystem proteinopathy: where myopathy and motor neuron disease converge. Muscle Nerve. 2021;63:442-54.

2. Leoni TB, Gonzalez-Salazar C, Rezende TJR, et al. A novel multisystem proteinopathy caused by a Missense ANXA11 variant. Ann Neurol. 2021;90:239-52.

3. Hill N, Frappier-Davignon L, Morrison B. The periodic health examination. Canadian Task Force on the Periodic Health Examination. Can Med Assoc J. 1979;121:1193-254.

4. Al-Obeidi E, Al-Tahan S, Surampalli A, et al. Genotype-phenotype study in patients with valosin-containing protein mutations associated with multisystem proteinopathy. Clin Genet. 2018;93:119-25.

5. Meyer H, Weihl CC. The VCP/p97 system at a glance: connecting cellular function to disease pathogenesis. J Cell Sci. 2014;127:3877-83.

6. van den Boom J, Meyer H. VCP/p97-mediated unfolding as a principle in protein homeostasis and signaling. Mol Cell. 2018;69:182-94.

7. Watts GD, Wymer J, Kovach MJ, et al. Inclusion body myopathy associated with Paget disease of bone and frontotemporal dementia is caused by mutant valosin-containing protein. Nat Genet. 2004;36:377-81.

8. Al-Tahan S, Al-Obeidi E, Yoshioka H, et al. Novel valosin-containing protein mutations associated with multisystem proteinopathy. Neuromuscul Disord. 2018;28:491-501.

9. Kim HJ, Kim NC, Wang YD, et al. Mutations in prion-like domains in hnRNPA2B1 and hnRNPA1 cause multisystem proteinopathy and ALS. Nature. 2013:495:467-73.

10. Bucelli RC, Arhzaouy K, Pestronk A, et al. SQSTM1 splice site mutation in distal myopathy with rimmed vacuoles. Neurology. 2015;85:665-74.

11. Taylor JP. Multisystem proteinopathy: intersecting genetics in muscle, bone, and brain degeneration. Neurology. 2015;85:658-60.

12. Lin KP, Tsai PC, Liao YC, et al. Mutational analysis of MATR3 in Taiwanese patients with amyotrophic lateral sclerosis. Neurobiol Aging. 2005;2015(36):e2001-4.

13. Mackenzie IR, Nicholson AM, Sarkar M, et al. TIA1 mutations in amyotrophic lateral sclerosis and frontotemporal dementia promote phase separation and alter stress granule dynamics. Neuron. 2017;95:808-816. e809.

14. Maruyama $\mathrm{H}$, Morino $\mathrm{H}$, Ito $\mathrm{H}$, et al. Mutations of optineurin in amyotrophic lateral sclerosis. Nature. 2010;465:223-6.

15. Albagha OM, Visconti MR, Alonso N, et al. Genome-wide association study identifies variants at CSF1, OPTN and TNFRSF11A as genetic risk factors for Paget's disease of bone. Nat Genet. 2010;42:520-4.

16. Scotto di Carlo F, Pazzaglia L, Esposito T, Gianfrancesco F. The loss of profilin 1 causes early onset Paget's disease of bone. J Bone Miner Res. 2020;35:1387-98.

17. Merlotti D, Materozzi M, Bianciardi S, et al. Mutation of PFN1 gene in an early onset, polyostotic Paget-like disease. J Clin Endocrinol Metab. 2020;105:2553-65.

18. Wu CH, Fallini C, Ticozzi N, et al. Mutations in the profilin 1 gene cause familial amyotrophic lateral sclerosis. Nature. 2012:488:499-503.

19. Weihl CC, Pestronk A, Kimonis VE. Valosin-containing protein disease: inclusion body myopathy with Paget's disease of the bone and frontotemporal dementia. Neuromuscul Disord. 2009;19:308-15.

20. Richards S, Aziz N, Bale S, et al. Standards and guidelines for the interpretation of sequence variants: a joint consensus recommendation of the American College of Medical Genetics and Genomics and the Association for Molecular Pathology. Genet Med. 2015;17:405-24.

21. Scuffham TM, MacMillan JC. Huntington disease: who seeks presymptomatic genetic testing, why and what are the outcomes? J Genet Couns. 2014;23:754-61. 
22. Crozier S, Robertson N, Dale M. The psychological impact of predictive genetic testing for Huntington's disease: a systematic review of the literature. J Genet Couns. 2015;24:29-39.

23. Tilleras KH, Kjoelaas SH, Dramstad E, Feragen KB, von der Lippe C. Psychological reactions to predictive genetic testing for Huntington's disease: a qualitative study. J Genet Couns. 2020;29:1093-105.

24. Mehta SG, Khare M, Ramani R, et al. Genotype-phenotype studies of VCPassociated inclusion body myopathy with Paget disease of bone and/or frontotemporal dementia. Clin Genet. 2013;83:422-31.

25. Bugiardini E, Morrow JM, Shah S, et al. The diagnostic value of MRI pattern recognition in distal myopathies. Front Neurol. 2018;9:456.

26. Evangelista T, Weihl CC, Kimonis V, Lochmuller H, Consortium VCPrd. 215th ENMC International Workshop VCP-related multi-system proteinopathy (IBMPFD) 13-15 November 2015, Heemskerk, The Netherlands. Neuromuscul Disord. 2016;26:535-47.

27. Kazamel M, Sorenson EJ, McEvoy KM, et al. Clinical spectrum of valosin containing protein (VCP)-opathy. Muscle Nerve. 2016;54:94-9.

28. Diaz-Manera J, Llauger J, Gallardo E, Illa I. Muscle MRI in muscular dystrophies. Acta Myol. 2015;34:95-108.

29. Weihl CC, Temiz P, Miller SE, et al. TDP-43 accumulation in inclusion body myopathy muscle suggests a common pathogenic mechanism with frontotemporal dementia. J Neurol Neurosurg Psychiatry. 2008;79:1186-9.

30. Figueroa-Bonaparte S, Hudson J, Barresi R, et al. Mutational spectrum and phenotypic variability of VCP-related neurological disease in the UK. J Neurol Neurosurg Psychiatry. 2016;87:680-1.

31. Llewellyn KJ, Nalbandian A, Weiss LN, et al. Myogenic differentiation of VCP disease-induced pluripotent stem cells: a novel platform for drug discovery. PLOS ONE. 2017;12:e0176919.

32. Zhang T, Mishra P, Hay BA, Chan D, Guo M. Valosin-containing protein (VCP/p97) inhibitors relieve mitofusin-dependent mitochondrial defects due to VCP disease mutants. Elife. 2017;6:e17834

33. Wang B, Maxwell BA, Joo JH, et al. ULK1 and ULK2 regulate stress granule disassembly through phosphorylation and activation of VCP/p97. Mol Cell. 2019;74:742-757.e748.

34. Kim EJ, Park YE, Kim DS, et al. Inclusion body myopathy with Paget disease of bone and frontotemporal dementia linked to VCP p.Arg155Cys in a Korean family. Arch Neurol. 2011;68:787-96.

35. Ducharme $\mathrm{S}$, Dols A, Laforce $\mathrm{R}$, et al. Recommendations to distinguish behavioural variant frontotemporal dementia from psychiatric disorders. Brain. 2020;143:1632-50.

36. Papadimas GK, Paraskevas GP, Zambelis T, et al. The multifaceted clinical presentation of VCP-proteinopathy in a Greek family. Acta Myol. 2017:36:203-6.

37. Hubbers CU, Clemen CS, Kesper K, et al. Pathological consequences of VCP mutations on human striated muscle. Brain. 2007;130:381-93.

38. Rascovsky K, Hodges JR, Knopman D, et al. Sensitivity of revised diagnostic criteria for the behavioural variant of frontotemporal dementia. Brain. 2011;134:2456-77.

39. Casaletto KB, Staffaroni AM, Wolf A, et al. Active lifestyles moderate clinical outcomes in autosomal dominant frontotemporal degeneration. Alzheimers Dement. 2020:16:91-105.

40. Kiousi V, Arnaoutoglou M, Printza A. Speech and language intervention for language impairment in patients in the FTD-ALS spectrum. Hell J NuCl Med. 2019;22(Suppl):133-46.

41. Tsai RM, Boxer AL. Treatment of frontotemporal dementia. Curr Treat Options Neurol. 2014;16:319.

42. Leroy M, Bertoux M, Skrobala E, et al. Characteristics and progression of patients with frontotemporal dementia in a regional memory clinic network. Alzheimers Res Ther. 2021;13:19.

43. Noufi P, Khoury R, Jeyakumar S, Grossberg GT. Use of cholinesterase inhibitors in non-Alzheimer's dementias. Drugs Aging. 2019;36:719-31.

44. Boxer AL, Knopman DS, Kaufer DI, et al. Memantine in patients with frontotemporal lobar degeneration: a multicentre, randomised, double-blind, placebo-controlled trial. Lancet Neurol. 2013;12:149-56.

45. van Staa TP, Selby P, Leufkens HG, Lyles K, Sprafka JM, Cooper C. Incidence and natural history of Paget's disease of bone in England and Wales. J Bone Miner Res. 2002;17:465-71.

46. Tan A, Ralston SH. Paget's disease of bone. QJM. 2014;107:865-9.

47. Ralston SH, Corral-Gudino L, Cooper C, et al. Diagnosis and management of Paget's disease of bone in adults: a clinical guideline. J Bone Miner Res. 2019:34:579-604.
48. Ralston SH. Bisphosphonates in the management of Paget's disease. Bone. 2020;138:115465.

49. Merlotti $D$, Rendina $D$, Muscariello $R$, et al. Preventive role of vitamin D supplementation for acute phase reaction after bisphosphonate infusion in Paget's disease. J Clin Endocrinol Metab. 2020;105:e466-76.

50. Cronin O, Forsyth L, Goodman K, et al. Zoledronate in the prevention of Paget's (ZiPP): protocol for a randomised trial of genetic testing and targeted zoledronic acid therapy to prevent SQSTM1-mediated Paget's disease of bone. BMJ Open. 2019;9:e030689.

51. Tan A, Goodman K, Walker A, et al. Long-term randomized trial of intensive versus symptomatic management in Paget's disease of bone: the PRISM-EZ study. J Bone Miner Res. 2017;32:1165-73.

52. Brooks BR, Miller RG, Swash M, Munsat TL, World Federation of Neurology Research Group on Motor Neuron D. El Escorial revisited: revised criteria for the diagnosis of amyotrophic lateral sclerosis. Amyotroph Lateral Scler Other Motor Neuron Disord. 2000;1:293-9.

53. de Carvalho M, Dengler R, Eisen A, et al. Electrodiagnostic criteria for diagnosis of ALS. Clin Neurophysiol. 2008;119:497-503.

54. Hannaford A, Pavey N, van den Bos M, et al. Diagnostic utility of gold coast criteria in amyotrophic lateral sclerosis. Ann Neurol. 2021;89:979-86.

55. Diagnosis ETFo, Management of Amyotrophic Lateral S, Andersen PM, et al. EFNS guidelines on the clinical management of amyotrophic lateral sclerosis (MALS) - revised report of an EFNS task force. Eur J Neurol. 2012;19:360-75.

56. Jerath NU, Crockett CD, Moore SA, et al. Rare manifestation of a C.290 C>T, p.Gly97Glu VCP mutation. Case Rep Genet. 2015;2015:239167.

57. Gonzalez MA, Feely SM, Speziani F, et al. A novel mutation in VCP causes Charcot-Marie-Tooth Type 2 disease. Brain. 2014;137:2897-902.

58. De Ridder W, Azmi A, Clemen CS, et al. Multisystem proteinopathy due to a homozygous p.Arg159His VCP mutation: a tale of the unexpected. Neurology. 2019;94:e785-96.

59. Postuma RB, Berg D, Stern M, et al. MDS clinical diagnostic criteria for Parkinson's disease. Mov Disord. 2015;30:1591-601.

60. Kimonis VE, Kovach MJ, Waggoner B, et al. Clinical and molecular studies in a unique family with autosomal dominant limb-girdle muscular dystrophy and Paget disease of bone. Genet Med. 2000;2:232-41.

61. Feingold B, Mahle WT, Auerbach S, et al. Management of cardiac involvement associated with neuromuscular diseases: a scientific statement from the American Heart Association. Circulation. 2017; 136:e200-31.

62. Kimonis VE, Fulchiero E, Vesa J, Watts G. VCP disease associated with myopathy, Paget disease of bone and frontotemporal dementia: review of a unique disorder. Biochim Biophys Acta. 2008;1782:744-8.

63. Wang SC, Smith CD, Lombardo DM, Kimonis V. Characteristics of VCP mutation-associated cardiomyopathy. Neuromuscul Disord. 2021:31:701-5.

64. Ikenaga C, Findlay AR, Seiffert M, et al. Phenotypic diversity in an international Cure VCP Disease registry. Orphanet J Rare Dis. 2020;15:267.

65. Plewa J, Surampalli A, Wencel M, et al. A cross-sectional analysis of clinical evaluation in 35 individuals with mutations of the valosin-containing protein gene. Neuromuscul Disord. 2018;28:778-86.

66. Finder JD, Birnkrant D, Carl J, et al. Respiratory care of the patient with Duchenne muscular dystrophy: ATS consensus statement. Am J Respir Crit Care Med. 2004;170:456-65.

67. Ragette R, Mellies U, Schwake C, Voit T, Teschler H. Patterns and predictors of sleep disordered breathing in primary myopathies. Thorax. 2002:57:724-8

68. Nalbandian A, Donkervoort S, Dec E, et al. The multiple faces of valosincontaining protein-associated diseases: inclusion body myopathy with Paget's disease of bone, frontotemporal dementia, and amyotrophic lateral sclerosis. J Mol Neurosci. 2011:45:522-31.

69. Nalbandian A, Nguyen C, Katheria V, et al. Exercise training reverses skeletal muscle atrophy in an experimental model of VCP disease. PLoS ONE. 2013;8:e76187

70. Kimonis V, Hamorsky K, Surampalli A, Wencel M, Khare M. The influence of diet and exercise on the physical health of affected individuals with VCP diseae. Int J Biotechnol Wellness Ind. 2014;3:46-52.

71. Kleim JA, Jones TA. Principles of experience-dependent neural plasticity: implications for rehabilitation after brain damage. J Speech Lang Hear Res. 2008:51:S225-39. 
72. Bogart KR, Dermody SS. Relationship of rare disorder latent clusters to anxiety and depression symptoms. Health Psychol. 2020;39:307-15.

73. A standard framework for levels of integrated healthcare and update throughout the document. Available at https://www.integration.samhsa. gov/integrated-care-models/A_Standard_Framework_for_Levels_of_ Integrated_Healthcare.pdf. Accessed 13 May 2020.

74. Roth JD, Pariser JJ, Stout TE, Misseri R, Elliott SP. Presentation and management patterns of lower urinary tract symptoms in adults due to rare inherited neuromuscular diseases. Urology. 2020;135:165-70.

75. Morse Cl, Higham K, Bostock EL, Jacques MF. Urinary incontinence in men with Duchenne and Becker muscular dystrophy. PLOS ONE. 2020;15:e0233527.

76. Petty RKH, Eugenicos MP, Hamilton MJ, et al. The prevalence of faecal incontinence in myotonic dystrophy type 1. Neuromuscul Disord. 2019;29:562-6.

77. Llewellyn KJ, Nalbandian A, Jung KM, et al. Lipid-enriched diet rescues lethality and slows down progression in a murine model of VCP-associated disease. Hum Mol Genet. 2014;23:1333-44.

78. Romagnolo DF, Selmin OI. Mediterranean diet and prevention of chronic diseases. Nutr Today. 2017;52:208-22.

\section{Publisher's Note}

Springer Nature remains neutral with regard to jurisdictional claims in published maps and institutional affiliations.

- fast, convenient online submission

- thorough peer review by experienced researchers in your field

- rapid publication on acceptance

- support for research data, including large and complex data types

- gold Open Access which fosters wider collaboration and increased citations

- maximum visibility for your research: over $100 \mathrm{M}$ website views per year

At BMC, research is always in progress.

Learn more biomedcentral.com/submissions 